\title{
Lessons from tough cases
}

\author{
Young-Kyun Kim, DDS, PhD \\ Editor-in-Chief of JKAOMS \\ Department of Oral and Maxillofacial Surgery, Section of Dentistry, Seoul National University Bundang Hospital, Seongnam, Korea
}

Even if patients are given proper treatment, inevitable or unexplained adverse medical events and various unanticipated problems may arise in clinical practice. Since all oral and maxillofacial surgeons inevitably encounter major or minor postoperative complications and medical disputes, they must have a strong ability to deal with it. Even though "the cause is unknown" or "obviously not the fault of the surgeons," he should not be held responsible for failing to explain and respond appropriately to patients, guardians, or lawyers. If a medical problem occurs due to a serious error such as malpractice, medical practitioners should bear full responsibility for it, but when a problem occurs inevitably or due to unknown causes, medical staff should not be held unduly accountable.

In the field of dental medicine, particularly oral and maxillofacial surgery not only performs treatment of various diseases but also is responsible for esthetic plastic reconstructive treatment. However, more attention should be paid to the fact that oral and maxillofacial surgeons provide treatment for human beings. For example, when medical services, such as plastic surgery, orthognathic surgery, and petit molding, are performed, is it possible to provide medical care that completely satisfies patients? The author is fully confident that none of the current medical practitioners, including scholars known as authorities, can deliver treatment that always completely satisfies patients. It is very often the case that even though surgeons have delivered the best or optimal treatment

\footnotetext{
Young-Kyun Kim

Department of Oral and Maxillofacial Surgery, Section of Dentistry, Seoul National University Bundang Hospital, 82 Gumi-ro 173beon-gil, Bundang-gu, Seongnam 13620, Korea

TEL: +82-31-787-7541

E-mail:kyk0505@snubh.org

ORCID: https://orcid.org/0000-0002-7268-3870
}

(c) This is an open-access article distributed under the terms of the Creative Commons Attribution Non-Commercial License (http://creativecommons.org/ licenses/by-nc/4.0/), which permits unrestricted non-commercial use, distribution, and reproduction in any medium, provided the original work is properly cited.

Copyright (C) 2022 The Korean Association of Oral and Maxillofacial Surgeons. from their point of view, patients are dissatisfied and make a complaint about medical treatment. On the contrary, in some cases, even though the treatment provided for patients is not perfect, the patients may be very satisfied with medical services. Oral and maxillofacial surgeons should place importance on solving patients' functional and psychological problems as well as esthetic improvement. For this purpose, it is considered most important for them to maintain a good relationship with their patients.

In this regard, the author intends to emphasize the following aspects. 1) SCI(E) articles, lectures by renowned speakers, and contents dealt with at academic meetings and various seminars should be regarded only as reference materials. There are many cases that cannot be directly applied to patient treatment in actual clinical practice. 2) Medical practitioners should establish their own solid concepts on treatment and make utmost efforts to deliver evidence-based practices in compliance with ethical principles in health care. 3) Private training programs may be very helpful for improving individual surgical skills, but it is recommended to avoid training programs that focus on surgical skills and techniques without providing lectures on complications or other problems and presenting solutions to them. 4) It should also be noted that oral and maxillofacial surgeons are supposed to treat patients as human beings rather than pretentiously showing off advanced and sophisticated surgical procedures. Medical practitioners cannot deliver 100\% perfect treatment not only in dentistry but also in any other field of medicine. What patients expect from dentists would not be just $100 \%$ perfect treatment, either. In a recent issue report published by the Korean Dental Association ${ }^{1}$, it was pointed out that schools of dentistry have been overly focused on perfectionoriented surgical techniques and treatment in the dental education of students. It is natural to strive to perform perfect dental treatment, but more importantly, it is most important to strengthen the relationship between the patient and the dentist and to treat patients so that they can trust the dentist. 
Oral and maxillofacial surgeons should learn lessons from tough cases and complications reported by other researchers to prevent inappropriate treatment. In addition, it is also suggested that they should look back on and carefully review various problems and complications that they have experienced in order to develop the ability to cope with similar cases when they are faced with them.

\section{Conflict of Interest}

No potential conflict of interest relevant to this article was reported.

\section{Reference}

1. Issue Report. Vol. 9. Dentist's stress causes and management strategies [Internet]. Seoul: Health Policy Institute; 2019 Aug 16 [cited 2022 Feb 18]. Available from: http://www.hpikda.or.kr/bbs/bbs list.asp?menuId=pub\&pageId=issue

How to cite this article: Kim YK. Lessons from tough cases.

J Korean Assoc Oral Maxillofac Surg 2022;48:1-2. https://doi. org/10.5125/jkaoms.2022.48.1.1 